Check for updates

Cite this: RSC Adv., 2021, 11, 10061

\section{Assembled small organic molecules for photodynamic therapy and photothermal therapy}

\author{
Lixin Sun,† Jian Wang, $\uparrow$ Baochan Yang, Xinxin Wang, Gengxiang Yang, Xiqian Wang, \\ Yuying Jiang, Tianyu Wang (D) * and Jianzhuang Jiang (DD
}

As a worldwide major public health problem, cancer is one of the leading causes of death. Effective treatment of cancer is an important challenge. Therefore, photodynamic therapy (PDT) and photothermal therapy (PTT) have been widely applied as anti-tumour strategies due to their highperformance and limited side effects. Inspired by natural supramolecular architectures, such as cytochromes and photosystems, the hierarchical supramolecular assembly of small organic molecules has been developed for their use as photosensitizers or photothermal agents for PDT and PTT, respectively. In this manuscript, we will summarize the recent progress of PDT and PTT based on the assembly of small organic molecules.
Received 22nd January 2021 Accepted 2nd March 2021

DOI: 10.1039/d1ra00579k

rsc.li/rsc-advances
PDT needs proper amount of oxygen while tumour tissue is usually hypoxic. ${ }^{\mathbf{1 4 1 5}}$ Moreover, the effects of PDT on the antitumour immune systems are also complex. ${ }^{16-18}$

To deal with these problems and difficulties, the first strategy that comes to mind can be the synergy of various treatments. For example, the combination of chemotherapy with PDT. ${ }^{\mathbf{1 9 , 2 0}}$ (PDT) has attracted increased attention ${ }^{2-7}$ The mechanism of PDT is dependent on photocatalytic reactive oxygen species (ROS) generation (Scheme 1A), which changes the mitochondrial membrane potential and promotes apoptosis of cancer cells. $^{\mathbf{8} 9}$ In the early days, PDT research was focused on the synthesis of new photosensitizers. ${ }^{\mathbf{1 0 - 1 2}}$ For example, porphyrin derivatives have been the most popular photosensitizers. Many porphyrin, chlorin, and phthalocyanine molecular systems have been developed as photosensitizers. ${ }^{\mathbf{1 0 - 1 2}}$ It is worth mentioning that most of the assemblies discussed in this article are also dependent on porphyrin derivatives, even though other dye molecules, such as BODIPY, were also involved. In general, the high-performance photosensitizers are expected to have strong absorbance at long wavelength, higher ROS quantum yield, lower dark toxicity, and better metabolic characteristics. ${ }^{\mathbf{1 0 , 1 2}}$ Notably, these requirements for high-performance photosensitizers are also contradictory to some extent. For example, although the long wavelengths light can pass through thicker tissues, their energy also can be too low for photocatalytic ROS generation., ${ }^{6,12,13}$ Nevertheless, synthesis of more efficient photosensitizer molecules has many difficulties, and tedious organic synthesis is more likely to push up the cost of PDT. In addition, PDT itself also has some limitations. For instance,

Beijing Key Laboratory for Science and Application of Functional Molecular and Crystalline Materials, Department of Chemistry, University of Science and Technology Beijing, Beijing 100083, China. E-mail: twang@ustb.edu.cn; jianzhuang@ustb.edu.cn

$\dagger$ These authors contributed equally.
(A)

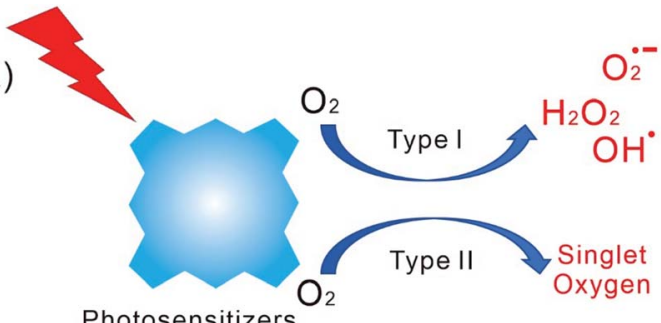

ROS

(B)

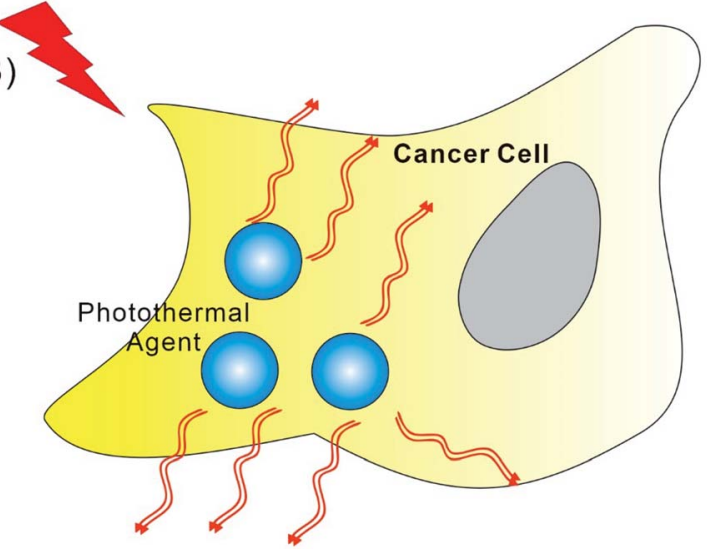

Scheme 1 Schematic illustration showing the mechanism of PDT (A) and the mechanism of PTT (B). 
For many research works discussed in the article, the use of combined chemotherapy was applied. Moreover, the strategy concerning nanomedicine was also introduced into PDT. ${ }^{21-23}$ Therefore, the development of PDT becomes increasingly dependent on the hierarchically supramolecular assembly of photosensitizers with different building blocks. ${ }^{24-28}$ For PDT, the supramolecular assembly of photosensitizers must play particularly important roles. This issue is not only due to the controlled release of photosensitizers, but also because of the demand for achieving the best photophysical performance. ${ }^{29-31}$ In fact, the photosensitive molecules, such as porphyrins, are quite common in organisms, however, these molecules rarely exist in a dispersed monomolecular state in cells. Instead, porphyrins are always enwrapped by different proteins in living system. The most famous architectures containing porphyrins can be photosystem, cytochrome, and haemoglobin. ${ }^{32-35}$ The functions of porphyrin derivatives within these complexes, such as absorbing light energy, electron transfer, and binding with small molecules, are dependent on the co-assembly of porphyrin with proteins.

Inspired by these natural supramolecular architectures, nanodrugs for PDT based on assembly have been developed. ${ }^{36-38}$ In this context, the supramolecular architectures for PDT with small amphiphilic molecules working as building blocks have obvious advantages. $^{24,25}$ Firstly, simple organic molecules induce the nanostructures and properties of assemblies with better controllability. ${ }^{39-41}$ Secondly, the safety and low-cost of many small organic molecules make their assemblies more feasible for PDT. Thirdly, developing supramolecular systems based on small amphiphilic building blocks can take advantage of the mass accumulation of photosensitizers synthesis for PDT research, which were achieved over the past few decades. In this manuscript, we will focus on the PDT based on the hierarchically supramolecular assembly of small organic molecules. We will summarize the progress in this field in the last five years. For the research works discussed in this article, the supramolecular structures of assembled small organic molecules are usually nanoparticles. Some gels systems with fibrous nanostructures were also involved. The cellular uptake of nanoparticles via the phagocytosis is a common approach. Certainly, the disassembly for release photosensitizers or other materials within the tissue or cells is involved.

On the other hand, since tumors are less resistant to heat than normal tissues, photothermal therapy (PTT) can be another important cancer therapy, which depends on converting light into thermal energy for thermal ablation of tumors by photothermal agents (Scheme 1B). ${ }^{\mathbf{4 2 , 4 3}}$ The main advantages of PTT are the less side effects and little drug resistant. Different from photosensitizers, there are varieties of materials that can be used as photothermal reagents, including Au nanorod, graphene, and $\mathrm{MoS}_{2}$ nanosheet. ${ }^{\mathbf{4}-46}$ Interestingly, the supramolecular assembly of organic photosensitizer, such as porphyrin, also can be applied as photothermal reagents. Therefore, with small organic dye molecules working as building blocks, photodynamic and photothermal synergistic cancer therapy can be achieved. The combined chemotherapy with PTT based on the supramolecular assemblies was also developed. These recent progresses will be addressed in this article. Overall, PDT and PTT based on the assembly of small organic molecules has attracted increased attentions recently. The corresponding research are becoming highly active in many countries, especially in China.

\section{Self-assembly of photosensitizers for PDT}

Porphyrin is a type of popular photosensitizer for PDT. The modification of porphyrin molecules could change their aggregation properties. Consequently, their supramolecular assemblies could show better properties for PDT. For example, Wang et al. have designed and synthesized an amphiphilic glycyrrhetinic acid-porphyrin conjugate (TPP-GA). The selfassembly of TPP-GA in water can form stable nanoparticles
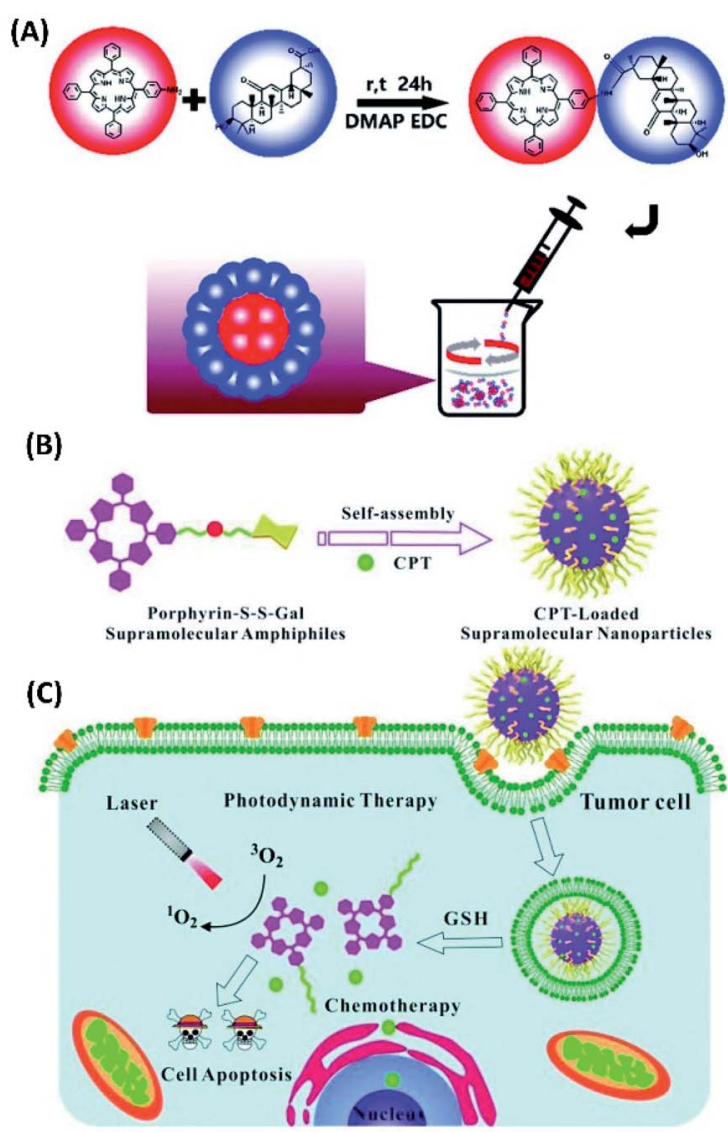

Fig. 1 (A) A schematic illustration of the synthesis of TPP-GA and the preparation of TPP-GA nanoparticles; ${ }^{47}$ (this figure has been reproduced from ref. 47 with permission from Royal Society of Chemistry). (B) Self-assembly of TPP-S-S-Gal and encapsulation of camptothecin (CPT) for PDT and chemotherapy; ${ }^{48}$ (this figure has been reproduced from ref. 48 with permission from Royal Society of Chemistry). (C) TPP-S-S-Gal/CPT micelles can be selectively taken up by cancer cells, CPT and photosensitizers could be released after disulfide bond cleavage by GSH. Under light irradiation, synergistic PDT and chemotherapy could lead to cell apoptosis ${ }^{48}$ (this figure has been reproduced from ref. 48 with permission from Royal Society of Chemistry). 
(Fig. 1A), which could be endocytosed easily by cancer cells. The in vitro experimental results suggest that TPP-GA nanoparticles show an efficient photodynamic therapeutic effect for cancer cells upon photo-irradiation. ${ }^{47}$

The amphiphilic photosensitizer was also synthesised by connecting galactose with TPP porphyrin via disulfide linkage (TPP-S-S-Gal). When TPP-S-S-Gal self-assembled into micelles, camptothecin (CPT), which is the chemotherapy drug for cancer, can be encapsulated in the hydrophobic core of micelles (Fig. 1B). These TPP-S-S-Gal/CPT micelles can be enriched in tumour cells. Since glutathione (GSH) molecules, which have free thiol, are relatively abundant in tumour cells, the interactions between TPP-S-S-Gal and GSH would induce disulfide bond cleavage and release of both photosensitizer porphyrin and chemotherapy drug CPT (Fig. 1C). Therefore, both PDT and chemotherapy can be performed. The in vitro cell experiments show that TPP-S-S-Gal micelles exhibited low dark toxicity and efficient integrative efficacy of PDT and chemotherapy towards A549 cells after light irradiation. ${ }^{48}$

To improve the cancer cell localization of photosensitizer chlorin e6, Kim et al. designed and synthesized cancer-targeting peptide p 18-4/chlorin e6 (Ce6)-conjugated polyhedral

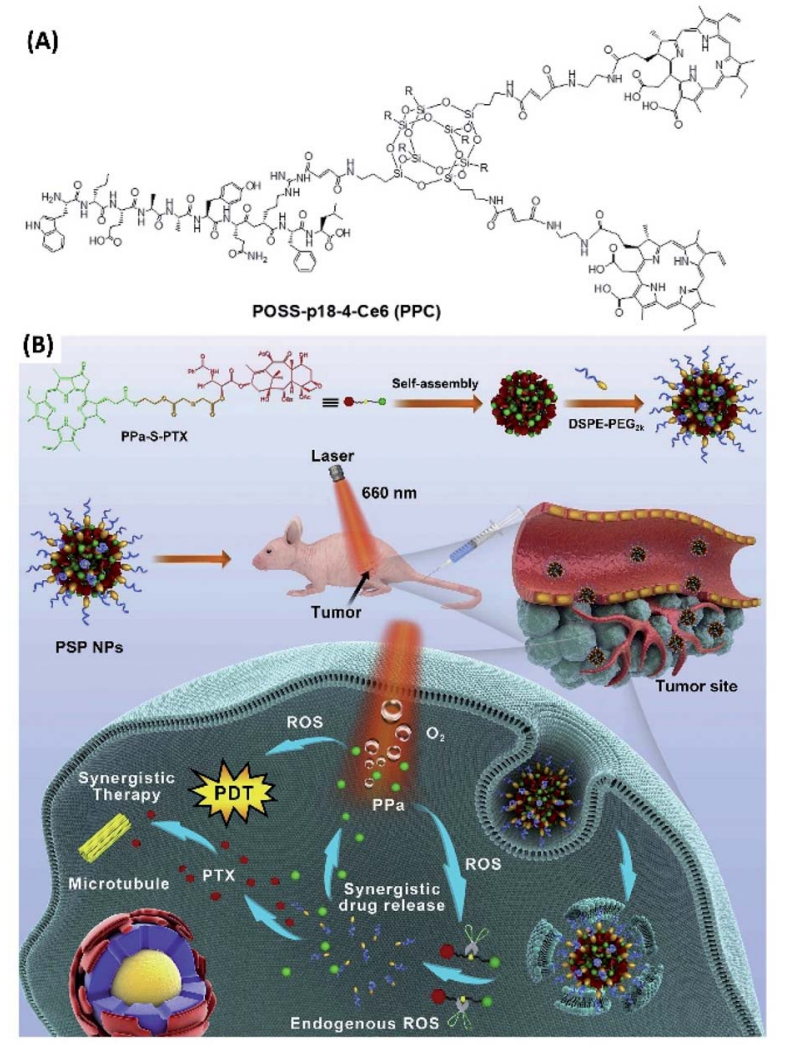

Fig. 2 (A) Molecular structure of peptide p 18-4/chlorin e6 (Ce6)conjugated polyhedral oligomeric silsesquioxane (PPC); ${ }^{49}$ (this figure has been reproduced from ref. 49 with permission from Elsevier). (B) The self-assembly of PPa-S-PTX with DSPE-PEG2k forms PEGylated nanoparticles. When these nanoparticles were delivered into the cancer cells, self-facilitated dual-synergistic multimodal cancer therapy could be achieved under laser irradiation ${ }^{50}$ (this figure has been reproduced from ref. 50 with permission from Elsevier). oligomeric silsesquioxane (PPC) nanoparticles (Fig. 2A). Compared with free Ce6, cellular uptake and targeting ability of PPC nanoparticles in breast cancer cell line MDA-MB-231 was largely enhanced. Moreover, PPC nanoparticles show significantly improved phototoxicity to MDA-MB-231 cells upon light irradiation, suggesting enhanced PDT efficacy. ${ }^{49}$

For the combination cancer therapy, the chemotherapy drug can also be directly connected with photosensitizer. Luo et al. designed and synthesized thioether bond-bridged heterotypic dimer (PPa-S-PTX) containing paclitaxel (PTX) and pyropheophorbide (PPa) (Fig. 2B). The assembly of PPa-S-PTX with ethylene glycol derivatives (1,2-distearoyl-sn-glycero-3-phosphoethanolamine- $N$-[methoxy(polyethyleneglycol)-2000], DSPEPEG2k) can form nanoparticles with good solubility in water. For the application of PPa-S-PTX nanoparticles, the multimodal cancer therapy can be achieved. Upon photoirradiation, the ROS generated by photosensitizer not only can be used for PDT, but also facilitate the cleavage of thioether bond for the release of paclitaxel for chemotherapy. Both in vitro and in vivo experiments suggest that pyropheophorbide-mediated PDT in combination with PTX-initiated chemotherapy exhibits synergistic antitumor activity. ${ }^{50}$

By connecting photosensitizers with short peptide, an approach based on combining photodynamic and immunological therapy was designed and developed. Thus, Song et al. synthesized the amphiphilic molecule (PpIX-1MT) containing protoporphyrin (PpIX) and 1-methyltryptophan (1MT) (Fig. 3A). $1 \mathrm{MT}$ is an enzyme inhibitor, which could effectively block the immunosuppressive enzyme (indoleamine 2,3-dioxygenase, IDO) and strengthen a durable immune response. For PpIX$1 \mathrm{MT}$, the hydrophobic components are palmitic acid and PpIX, while the hydrophilic poly(ethylene glycol) (PEG) segment is used as a linker for connecting porphyrin with a caspaseresponsive peptide sequence (Asp-Glu-Val-Asp, DEVD) and $1 \mathrm{MT}$. The self-assembly of PpIX-1MT can form nanoparticles, which can accumulate in tumor areas.

Both in vitro and in vivo experiments were performed for study the immunotherapy as well as PDT of PpIX-1MT nanoparticles for tumors. Under $630 \mathrm{~nm}$ light irradiation, porphyrin catalyse the changing of oxygen into ROS for PDT. While the apoptosis of cancer cells facilitated the expression of caspase-3 and the production of tumor antigens, which could trigger the immune response. And the immune system could be strengthened via the caspase-3, which induces the release of 1MT from PpIX-1MT. The results show that the cascaded synergistic effect based on PpIX-1MT nanoparticles inhibit both primary and lung metastasis tumor effectively (Fig. 3A and B). ${ }^{51}$

Another approach by combining photodynamic and immunological therapy was developed by Cheng et al. The amphiphilic molecule (PpIX-PEG8-KVPRNQDWL) was synthesized by connecting peptide with porphyrin via a PEG linker (Fig. 3C). PpIX-PEG8-KVPRNQDWL was found to self-assemble into nanoparticles, which was applied for PDT amplified immunotherapy against malignant melanoma. Under light irradiation, porphyrin-mediated PDT could induce cell apoptosis and/or necrosis. Meanwhile, not only the corresponding ROS also can stimulate the anti-tumor immune response, the melanoma 


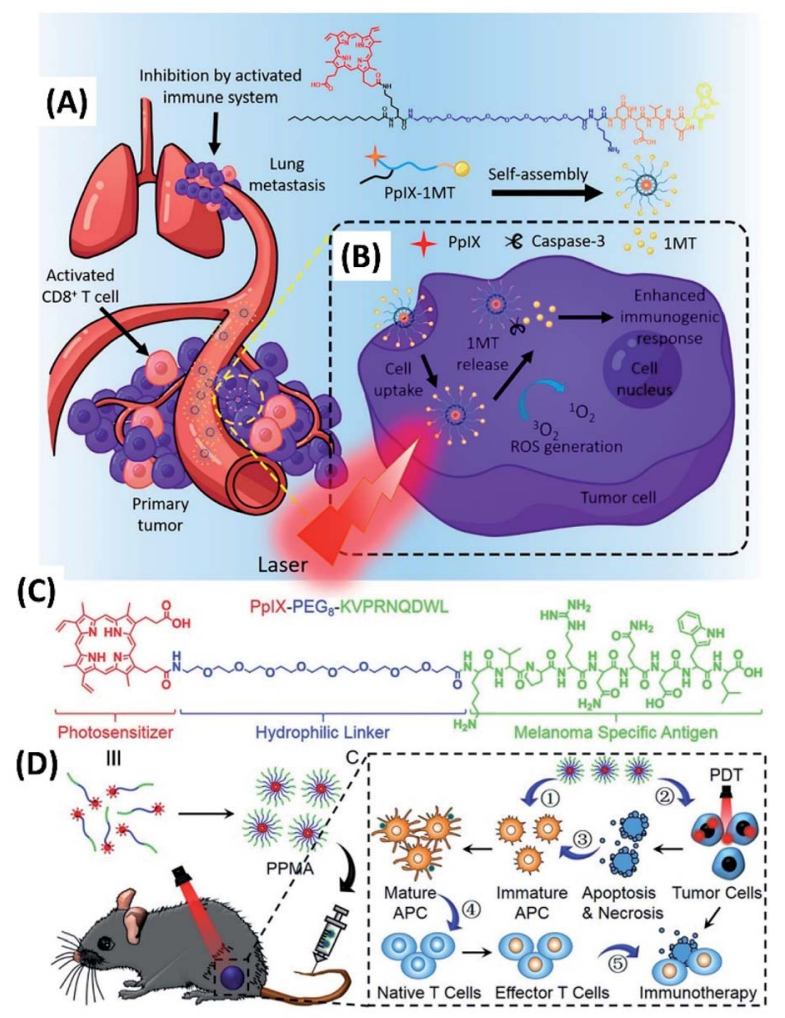

Fig. 3 (A) Molecular structure of PpIX-1MT. The PpIX-1MT nanoparticles could accumulate in the tumor areas; ${ }^{51}$ (this figure has been reproduced from ref. 51 with permission from American Chemical Society). (B) The cascaded synergistic effect based on PpIX-1MT nanoparticles; ${ }^{51}$ (this figure has been reproduced from ref. 51 with permission from American Chemical Society). (C) Molecular structure of PpIX-PEG8-KVPRNQDWL;52 (this figure has been reproduced from ref. 52 with permission from WILEY-VCH). (D) The mechanism of combined photodynamic immunotherapy by the self-assembly of PpIX-PEG8-KVPRNQDWL ${ }^{52}$ (this figure has been reproduced from ref. 52 with permission from WILEY-VCH).

specific antigen (KVPRNQDWL) peptide within the amphiphilic molecule can also activate the specific cytotoxic $\mathrm{T}$ cells for antitumor immunity (Fig. 3D). Both in vitro and in vivo experimental results suggest that the combined photodynamic immunotherapy exhibits significantly enhanced inhibition of melanoma growth. ${ }^{52}$

By connecting donner rhodamine B and acceptor protoporphyrin IX with PEG chains, the amphiphilic compound (RPP) with FRET (fluorescence resonance energy transfer) properties was synthesized (Fig. 4A). The self-assembly of RPP can form micelles, whose fluorescence colour varies with the $\mathrm{pH}$ value of living cells. Therefore, ratiometric $\mathrm{pH}$ imaging-guided PDT based on FRET can be achieved..$^{53}$

The changes of molecular structures could induce the variation supramolecular assemblies, which could give enhanced PDT effects. For example, Yu and his co-workers designed and synthesized pentapeptide FF-Amp-FF (AmpF) containing a central 4-amino-proline (Amp), as well as the FF-Amp-FF derivative by connecting a photosensitizer chlorin e6 (AmpFC) (Fig. 4B). The pH-sensitive cis/trans isomerization of Amp
(A)

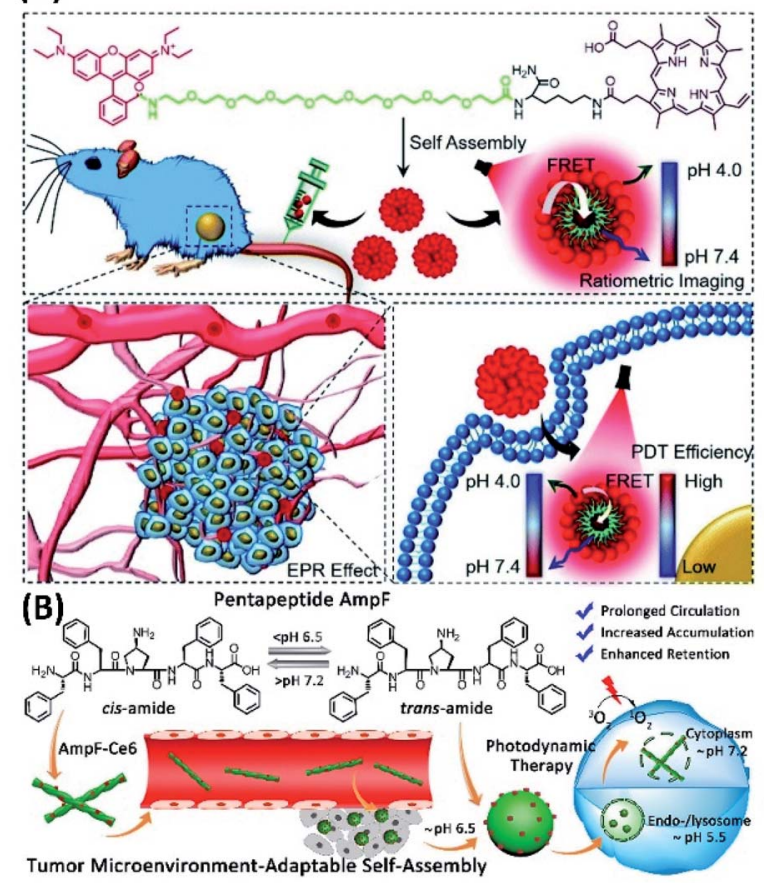

Fig. 4 (A) Molecular structure of the amphiphilic compound (RPP) for ratiometric $\mathrm{pH}$ sensing and tumour-targeted PDT; ${ }^{33}$ (this figure has been reproduced from ref. 53 with permission from Royal Society of Chemistry). (B) Proline isomerization-regulated tumour microenvironment-adaptable self-assembly of peptides for enhanced therapeutic efficacy ${ }^{54}$ (this figure has been reproduced from ref. 54 with permission from American Chemical Society).

amide bonds could induce the reversible self-assembly of FFAmp-FF into superhelices and nanoparticles, depending on neutral and mild acidic conditions. Therefore, the assembly of AmpF-C with AmpF could create tumor microenvironmentadaptable self-assembly systems (TMAS), which exhibit enhanced therapeutic efficacy. For the PDT application of AmpF/AmpF-C assemblies, the superhelices could cause enhanced intracellular ROS comparing with that of nanoparticles. Moreover, the reversible morphological transition between superhelices and nanoparticles can be adaptable to the $\mathrm{pH}$ gradient present in the endo/lysosome-mediated cellular uptake pathway, which could prolong circulation in animal body and improve accumulation and retention at tumor sites. ${ }^{54}$

\section{PDT based on co-assembly}

For developing new nanodrugs for PDT, the supramolecular coassembly represent the most important strategy. Thus, upon coassembly with some amphiphilic chiral molecules, such as peptide, phospholipid, and cholesterol derivatives, simply photosensitizers can be given much higher performance for PDT. Moreover, in the case of synergy of various treatments for cancer, the compounds for chemotherapy or immunotherapy also can be easily included by multicomponent co-assembly. For example, to increase the water solubility and to prevent the aggregation of photosensitizers for PDT, the 
supramolecular complexes between chlorophyll $a$ and cyclodextrins derivatives were developed. Among different coassemblies, the complex between chlorophyll $a$ and 2-hydroxypropyl- $\beta$-cyclodextrin (2-HP- $\beta$-CD) exhibited no dark toxicity and a high phototoxicity toward HT-29 cells inducing cell death via necrotic mechanism. Thus, complexes between chlorophyll $a$ and cyclodextrins derivatives could be a promising and potential formulation for applications in PDT (Fig. 5A). ${ }^{55}$

Co-assembly with short peptides not only can help carry hydrophobic photosensitizers into biological systems, but also can prevent the aggregation of these photosensitizers. Thus, the much better photophysical properties can be expected. Li et al. developed nanoparticles (nanoPSs) based on co-assembly of the aromatic peptide Fmoc-L3-OMe with meso-tetra( $p$-hydroxyphenyl)porphine ( $m$-THPP) (Fig. 5B). For the PDT applications of nanoPSs, the good separation and release of porphyrin renders not only good photodynamic properties, but also realtime fluorescence tracking property. ${ }^{56}$ Moreover, some porphyrin molecules on the surface of nanoPSs can be partially charged. Since the surface charges of the nanoparticles can accelerate the diffusion of gas molecules. These charged nanoPSs could facilitate the production and diffusion of singlet oxygen. Both the in vitro and in vivo experiments suggest that the nanoPSs show enhanced PDT efficacy by leading to complete tumour eradication.

Most interestingly, these nanoparticles show strong fluorescence upon excited by $850 \mathrm{~nm}$ two-photon laser. In contrast, only very weak two-photon fluorescence can be detected from

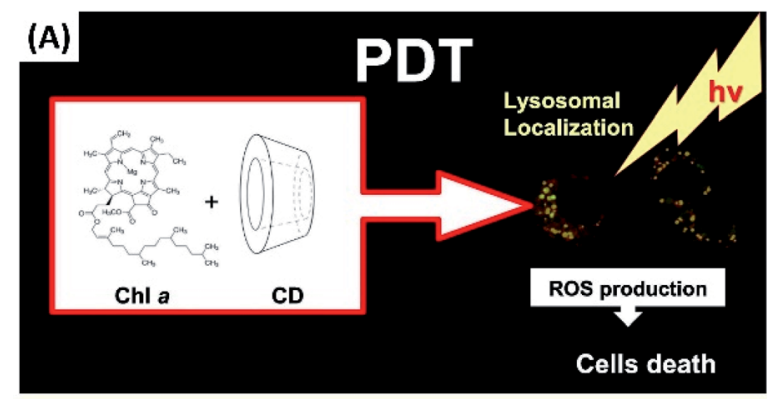

$(B)_{P}$

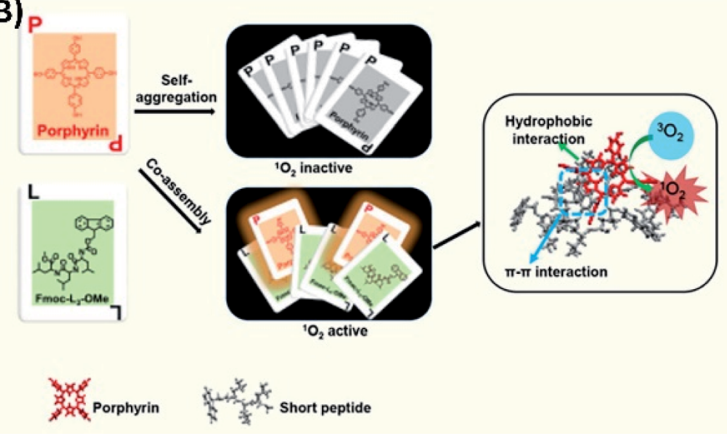

Fig. 5 (A) Chlorophyll a in cyclodextrin supramolecular complexes for PDT, ${ }^{55}$ (this figure has been reproduced from ref. 55 with permission from Elsevier). (B) The co-assembly between porphyrin and short peptides forms nanoparticles for PDT with real-time tracking property $^{56}$ (this figure has been reproduced from ref. 56 with permission from American Chemical Society). the pure $m$-THPP. The two-photon absorption ability of the nanoparticles induces effective generation of singlet oxygen, which lead the apoptosis of cancer cells (Fig. 6A). ${ }^{57}$

Although nanoparticles, which can be the supramolecular assembly of photosensitizers, are usually used for PDT, the hydrogels with fibrous nanostructures were also studied for carrying drugs for PDT. Yan et al. designed and synthesized bola-dipeptide by connecting two diphenylamine with long hydrophobic chain. This bola type molecule can form injectable hydrogels with good stability, rapid recovery, and excellent biocompatibility. By using the hydrogels as the drug carriers, 5aminolevulinic acid (5-ALA), which is prodrug for PDT by converting to PpIX through a heme biosynthetic pathway, can be encapsulated and further released in tumours (Fig. 6B). And the in situ biosynthetic formation of the photosensitizer can be utilized for efficient PDT. ${ }^{58}$

Ergosterol is a type of steroid molecule with anticancer activity. Both the self-assembly of ergosterol and the coassembly with chlorin e6 can form nanoparticles with biocompatibility and biodegradability. Therefore, the combined antitumor therapy applications of the ergosterol/Ce6 nanoparticles can be expected, depending on the anticancer activity of ergosterol, as well as the PDT from chlorin e6. The ergosterol/ Ce6 nanoparticles have improved stability, water solubility, excellent tumour targeting ability and prolonged blood circulation, as well as higher ROS generation by promoting type I photoreactions (Fig. 7A). In vitro cell experiments show ergosterol/Ce6 nanoparticles have remarkably phototoxicity upon light irradiation. For the in vivo experiments, the anticancer efficiency of ergosterol/Ce6 nanoparticles can be as high as $86.4 \%$, which is much higher than that of ergosterol

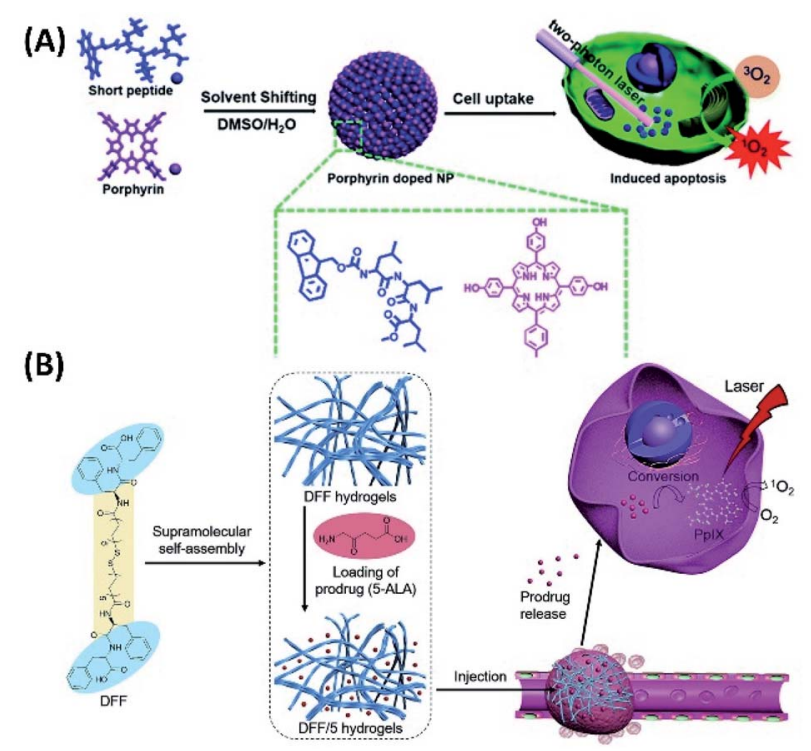

Fig. 6 (A) The co-assembly between porphyrin and short peptides forms nanoparticles for two-photon PDT; ${ }^{57}$ (this figure has been reproduced from ref. 57 with permission from Royal Society of Chemistry). (B) Bola-dipeptide-based injectable hydrogels for 5-ALA delivery and enhanced $\mathrm{PDT}^{58}$ (this figure has been reproduced from ref. 58 with permission from Elsevier). 


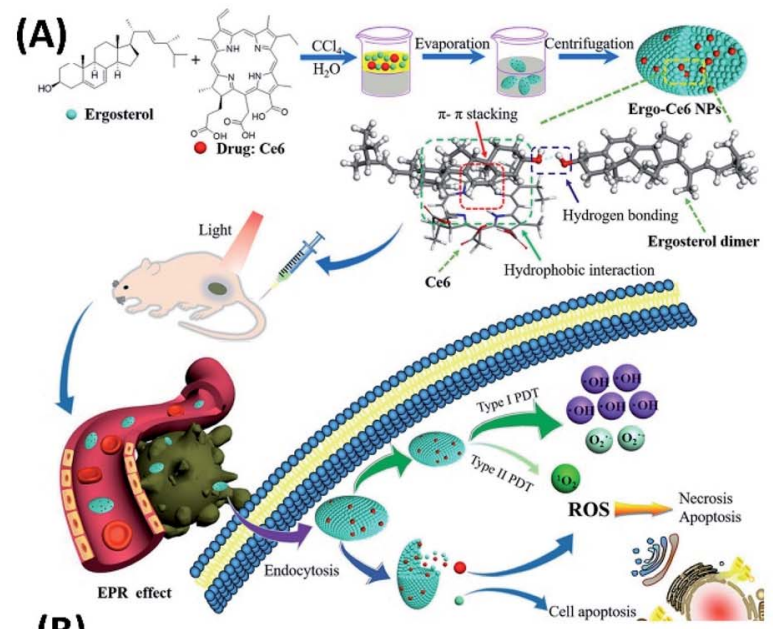

(B)
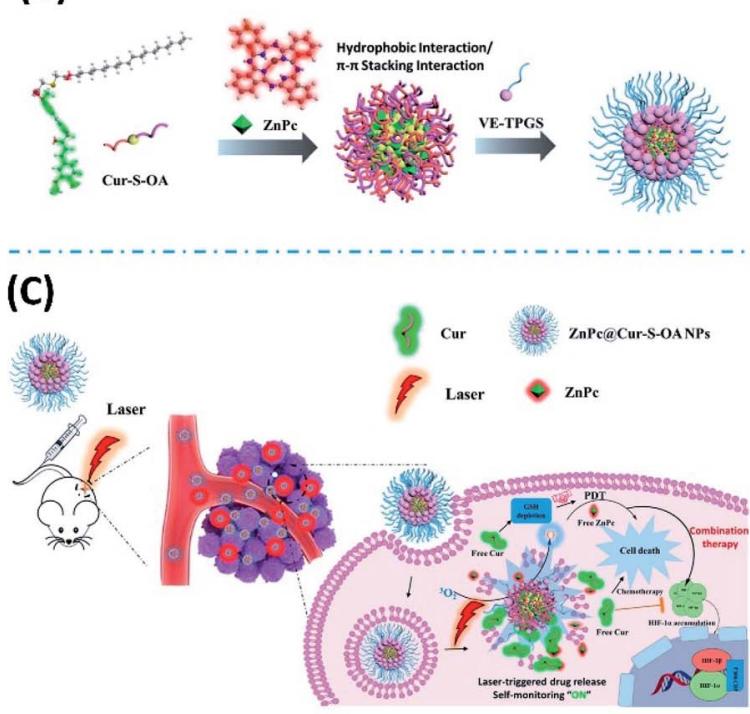

Fig. 7 (A) The co-assembled ergosterol/Ce6 nanoparticles can be used for combined antitumor therapy, depending on the anticancer activity of ergosterol, as well as the PDT from Ce6; ${ }^{59}$ (this figure has been reproduced from ref. 59 with permission from American Chemical Society). (B) The co-assembly of Cur-S-OA with zinc phthalocyanine (ZnPc) and vitamin E polyethylene glycol succinate (VE-TPGS) forms nanoparticles; ${ }^{60}$ (this figure has been reproduced from ref. 60 with permission from American Chemical Society). (C) ZnPcaCur-S-OA nanoparticles can be used for chemo-photodynamic combination therapy ${ }^{60}$ (this figure has been reproduced from ref. 60 with permission from American Chemical Society).

nanoparticles (51.0\%) or Ce6 PDT alone (59.5\%). ${ }^{59}$ Combined chemotherapy with PDT shows great potential for clinical application. While supramolecular assembly offers more possibilities for performing combined therapy.

For the application of PDT, the possible drawback can be from the high concentration of glutathione (GSH) in cancer cells, which could neutralize the generated ROS during PDT. Moreover, possible tissue hypoxia is another uncertain factor. The hypoxia-induced elevated hypoxia-inducible factor- $1 \alpha$ (HIF$1 \alpha$ ) could desensitize tumors to PDT. Considering curcumin has the anticancer ability with downregulating HIF-1 $\alpha$ and depleting GSH capability, Qu et al. synthesized the amphiphilic derivatives of curcumin (Cur-S-OA), and prepared the nanoparticles upon the co-assembly of Cur-S-OA with zinc phthalocyanine $(\mathrm{ZnPc})$ and vitamin E polyethylene glycol succinate (VETPGS) (Fig. 7B). Both in vitro and in vivo experiments suggest that the combined therapy nanoparticles show improved PDT efficacy due to downregulation of HIF- $1 \alpha$ and depletion of GSH. During this combined cancer therapy, the distribution of nanoparticles can be followed by detecting red fluorescence of ZnPc. Notably, the green fluorescence of curcumin moieties showed an "OFF-ON" activation, which enables additional imaging and real-time self-monitoring capabilities (Fig. 7C). ${ }^{60}$

Supramolecular hydrogels have significant advantages in drug delivery for PDT. Upon including different drugs into the hydrogels, combined chemotherapy with PDT can be achieved. Moreover, when the supramolecular gelators were designed with stimuli-responsive properties, better drug delivery can be expected. Pu et al. designed and synthesized the supramolecular gelator containing thioketal substituent, which can form

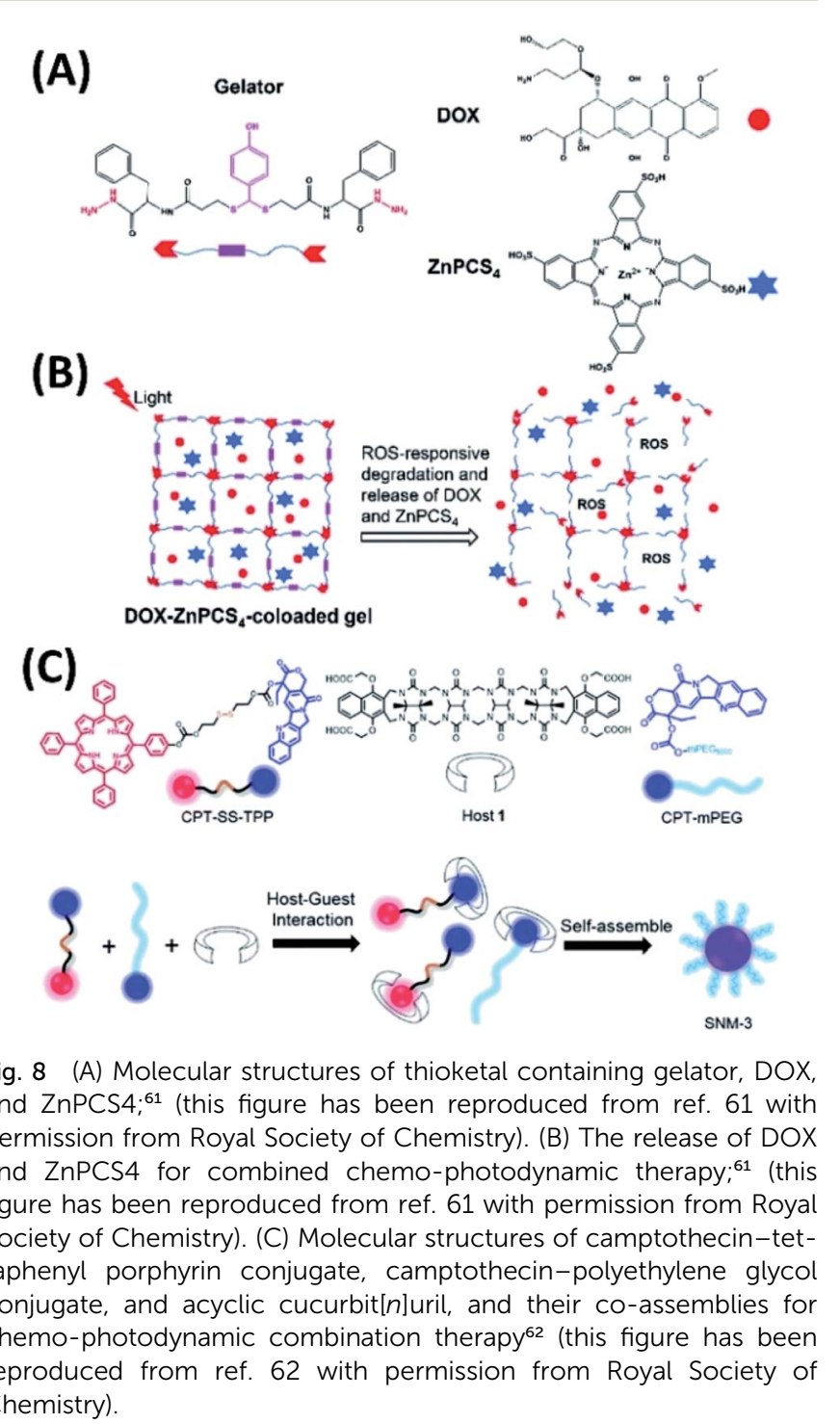


(A)

injectable supramolecular gels with ROS-responsive properties. Based on this injectable supramolecular gels, combined chemophotodynamic therapy system was developed by loading anticancer drug, doxorubicin hydrochloride (DOX), and photosensitizer, Zn(II) phthalocyanine tetrasulfonic acid (ZnPCS4) (Fig. 8A and B). The results from in vitro and in vivo experiments revealed that the injectable ROS-responsive DOX-ZnPCS4coloaded supramolecular gels exhibited excellent antitumor efficacy by a synergistic therapy. ${ }^{61}$

Another combined chemo-photodynamic therapy system was developed by Ma et al. as supramolecular nanomedicine. In this system, three component co-assembly containing a camptothecin-tetraphenyl porphyrin conjugate, camptothecin-polyethylene glycol conjugate, and acyclic cucurbit[n]uril was fabricated (Fig. 8C). Good efficacy of chemo-photodynamic combination therapy against cancer cell lines was achieved by varying the composition of different components. ${ }^{62}$

New combined chemo-photodynamic therapy system can also be developed via carrier-free strategy by the co-assembly of photosensitizer with chemotherapy drugs. In this context, Yan et al. have developed simple and green approach depending on the assembly of chlorine e6 (Ce6) with doxorubicin (DOX) (Fig. 9A). The Ce6/DOX co-assemblies are nanoparticles with average diameters of $70 \mathrm{~nm}$ and negative surface charge of $-20 \mathrm{mV}$. Compared with free Ce6 solution, Ce6/DOX nanoparticles tend to accumulate at the tumor region depending on potential enhanced permeability and retention (EPR) effect. These Ce6/DOX nanoparticles, which are safe for healthy tissue, can eventually eradicate the tumor during one treatment cycle in vivo. ${ }^{63}$

Similar system based on the co-assembly of photosensitizer with chemotherapy drugs was developed by Chen $e$ t al. thus, the assembly of tetra sodium mesotetra (sulfonatophenyl)porphyrin $\left(\mathrm{H}_{2}\right.$ TPPS) with doxorubicin (DOX) can form H2TPPS@DOX nanoparticles. Upon light irradiation, this type of nanoparticle can effectively reverse multidrug resistance of tumor cells and induce apoptosis of cancer cells. ${ }^{64}$

Certainly, the synergistic therapy based on the co-assembly of photosensitizer with other anticancer drugs is attracting increased interests. However, there may be significant differences in the way to carry out this idea. For example, to avoid some side effects, low-dose PDT is applied. Sun et al., synthesized ROS-responsive PEGylated hyperbranched polyphosphate containing thioketal linkers (RHPPE). The co-assembly of RHPPE with Ce6 and sorafenib forms ROS-responsive nanoparticles. Upon light irradiation, the disaggregation of nanoparticles, which is induced by the ROS produced by Ce6, could result in boosted sorafenib cascade release (Fig. 9B). The cascade-amplifying antitumor effects can be achieved from the

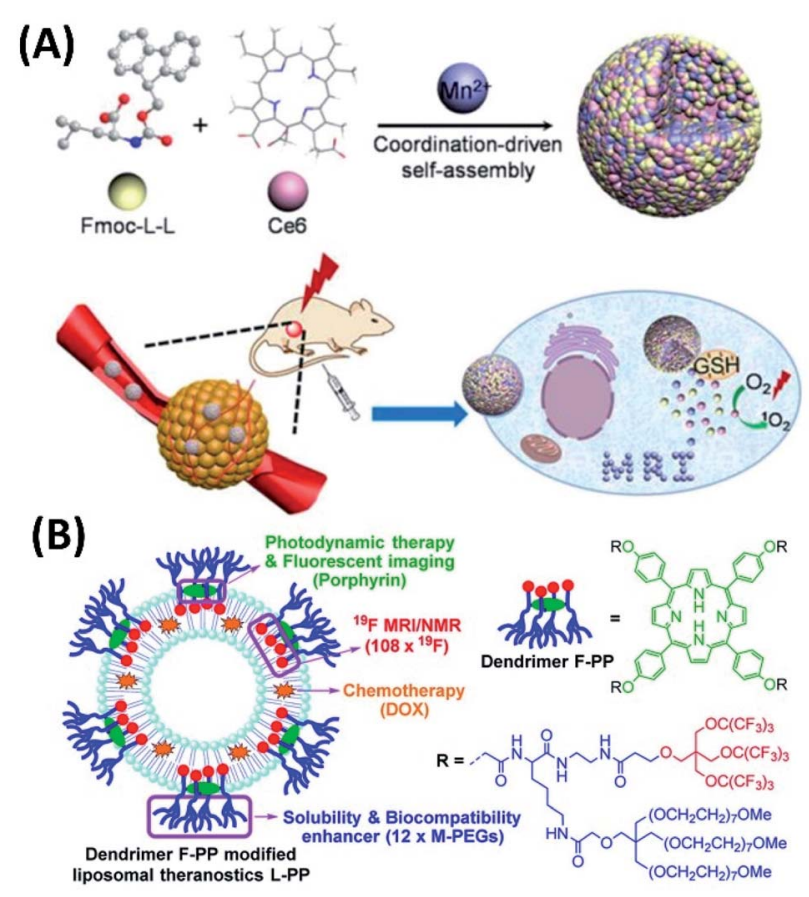

Fig. 10 (A) Fmoc-L-L/Mn ${ }^{2+} / \mathrm{Ce} 6$ co-assembly for magnetic resonance imaging-guided tumor PDT; 66 (this figure has been reproduced from ref. 66 with permission from American Chemical Society). (B) Fluorinated porphyrin-based theranostics for dual imaging and chemophotodynamic therapy ${ }^{67}$ (this figure has been reproduced from ref. 67 with permission from Royal Society of Chemistry). 

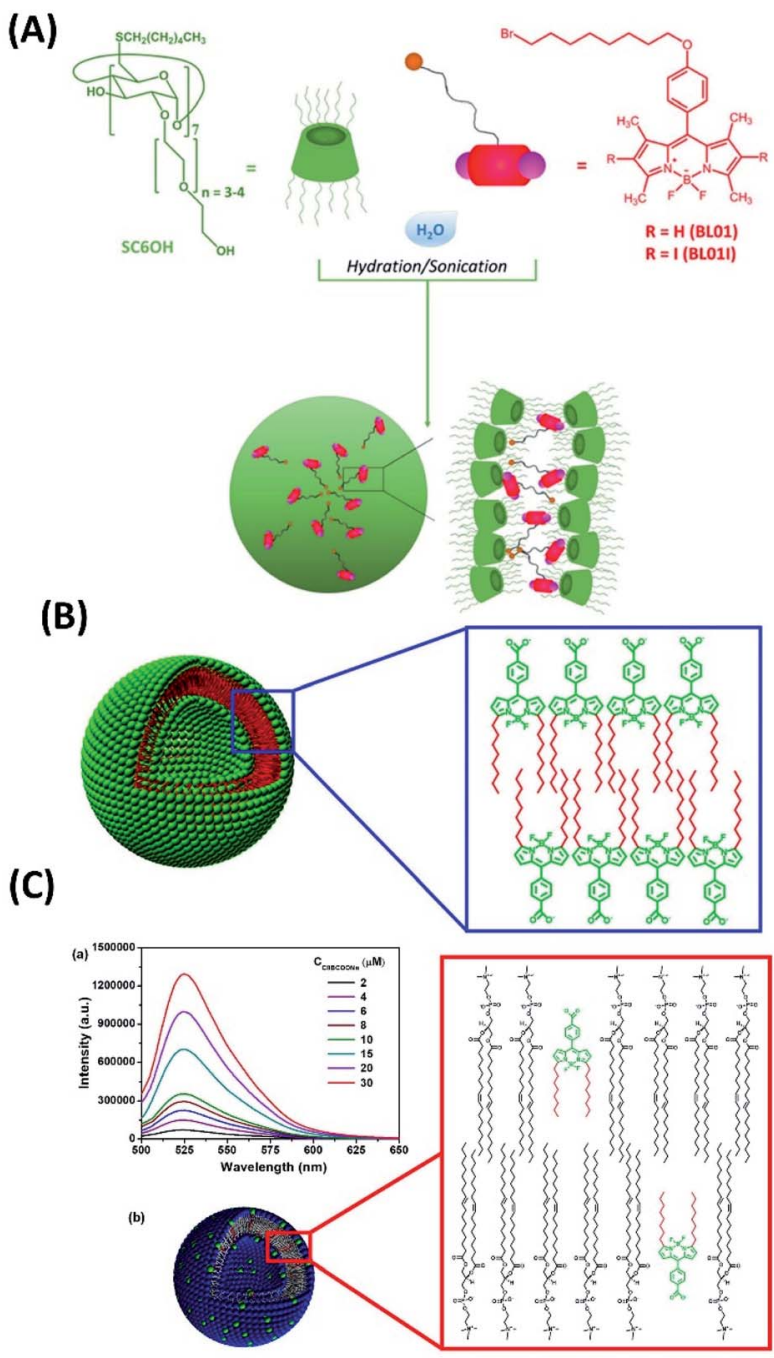

Fig. 11 (A) The co-assemblies between amphiphilic cyclodextrins with a halo-alkyl tailored iodinated BODIPY; ${ }^{68}$ (this figure has been reproduced from ref. 68 with permission from American Chemical Society). (B and C) The self-assembly of amphiphilic BODIPY as well as DOPC/ BODIPY co-assembly form vesicles with strong fluorescence intensity ${ }^{69}$ (this figure has been reproduced from ref. 69 with permission from American Chemical Society).

release of sorafenib, low-dose PDT and subsequently systemic antitumor immune responses. ${ }^{65}$

Including simple metal ions into co-assemblies could greatly enhance the corresponding combined treatments. For example, the co-assembly of 9-fluorenylmethyloxycarbonyl-L-leucine (Fmoc-L-L) with ionic manganese $\left(\mathrm{Mn}^{2+}\right)$, as well as Ce6 forms biometal-organic nanoparticles, which exhibit a high drug loading capability, inherent good biocompatibility, robust stability, and smart disassembly in response to glutathione (GSH). Upon the competitive coordination of GSH with $\mathrm{Mn}^{2+}$, the enhanced PDT can be achieved, not only due to the efficient delivery of the photosensitizer, but also because of the improved reductive tumor microenvironment. Most interestingly, the PDT in vivo can be monitored and evaluated by MRI through the long-term intracellular biochelation of $\mathrm{Mn}^{2+}$ (Fig. 10A). ${ }^{66}$
The combined cancer theranostics were also developed based on the co-assembly of dendrimeric porphyrin. Jiang et al. synthesized the amphiphilic porphyrin containing fluorinated dendrimer and PEG dendrimer (F-PP). For the application of FPP for cancer diagnosis and therapy, F-PP can be used for PDT, fluorescent imaging, as well as ${ }^{19} \mathrm{~F}$ magnetic resonance imaging (MRI). The co-assembly of F-PP with liposomes and chemotherapy drug doxorubicin (DOX) forms novel liposomal drug delivery systems, which show dual modal fluorescence $/{ }^{19} \mathrm{~F}$ MRI imaging and efficient chemo-photodynamic therapy (Fig. 10B). ${ }^{67}$

For the co-assemblies developed for PDT and different combined therapy systems, different kinds of photosensitizers can be involved. For example, the co-assemblies based on boron-dipyrromethenes (BODIPY), which is a type of powerful nonporphyrin photosensitizer, were also investigated recently. A. Mazzaglia et al. studied the co-assemblies between amphiphilic cyclodextrins (aCDs) with a halo-alkyl tailored iodinated BODIPY (Fig. 11A). ${ }^{68}$ These assemblies have good singlet oxygen production with higher photostability for PDT application. Another wonderful research work about BODIPY co-assemblies were developed by Wang et al. The amphiphilic BODIPY derivative (C8BCOONa) was synthesized. ${ }^{69}$ Both the self-assembly of C8BCOONa and the co-assembly with DOPC liposomes can form vesicles with extraordinarily strong fluorescence intensity (Fig. 11B and C). C8BCOONa co-assemblies have great potential for cell membrane imaging and PDT.

\section{Self-assembly of small organic molecules for PTT}

For PTT, although different materials can be used as photothermal reagents, the PTT based on the assembly of small amphiphilic molecules opens more possibilities. Yan et al. synthesized peptide-porphyrin conjugate (TPP-G-FF) by connecting dipeptide (L-phenylalanine-L-phenylalanine, $\mathrm{FF}$ ) with TPP porphyrin. By mimicking the biological organization of polypeptides and porphyrins, the self-assembly of TPP-G-FF can form nanodots, which introduce the $J$-aggregation of the porphyrin moieties. Interestingly, the self-assembly of TPP-G-FF totally inhibited fluorescence emission and singlet oxygen production from the porphyrin. Instead, the high light-to-heat conversion efficiency of the TPP-G-FF nanodots was achieved. The TPP-G-FF nanodots can be used for photothermal acoustic imaging and efficient antitumor therapy in vitro and in vivo (Fig. 12A). Notably, the peptide moieties nicely controlled the aggregation of porphyrin rings (Fig. 12B), which not only provide aqueous stability and biocompatibility of nanodots, but also lead to high light-to-heat conversion efficiency up to $54.2 \% .^{70}$

The aggregation enhanced PTT was also achieved by the assemblies of some hemicyanine dye derivatives. Zhang et al. synthesized mitochondria-targeted amphiphilic molecules containing hemicyanine dye, lipophilic triphenylphosphonium cation and phosphate ester group (NIR FL/PA). The NIR FL/PA can be activated through dephosphorylation by tumor-derived 
(A)

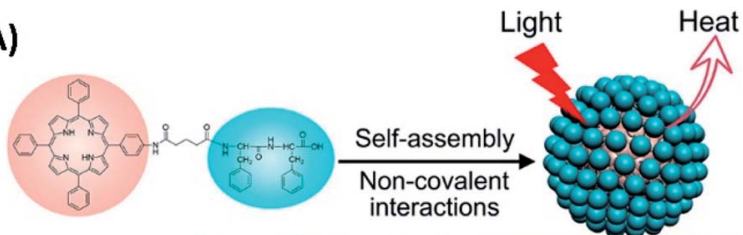

(B)

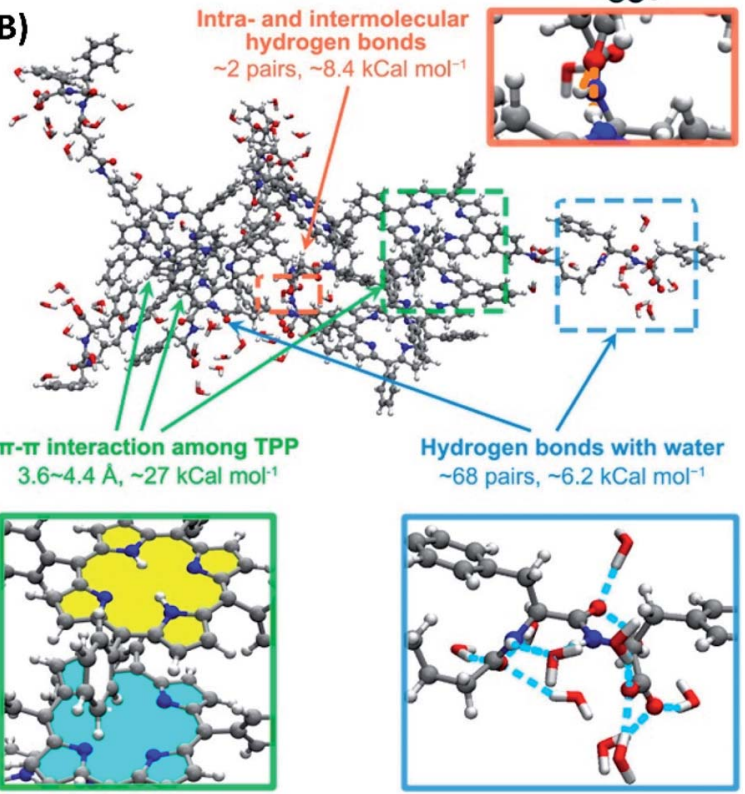

Fig. 12 (A) The self-assembly of peptide-porphyrin conjugates (TPPG-FF) forms nanodots for photothermal antitumor therapy; (this figure has been reproduced from ref. 70 with permission from American Chemical Society). (B) MD simulation of the self-assembled TPPG-FF molecules $^{70}$ (this figure has been reproduced from ref. 70 with permission from American Chemical Society).

alkaline phosphatase (ALP) with the changes of UV-Vis and fluorescence spectra. Since ALP is over-expressed in metastatic prostate cancer, NIR FL/PA can be used as near-infrared fluorescent/photoacoustic probe. Most importantly, the in situ self-assemble of activated NIR FL/PA show enhanced photothermal conversion for PTT (Fig. 13). ${ }^{71}$

\section{PTT based on co-assembly}

For PTT based on co-assemblies, Xie et al. developed novel PTT system by including dye molecules in metal-organic framework (MOF). Thus, the NIR dye cyanine (Cy) was embedded into the zeolitic imidazolate framework-8 to form nanoparticles (Cy@ZIF-8). The Cy@ZIF-8 nanoparticles have good NIR absorbance and photothermal conversion efficiency, with improved water solubility and photostability. Both the in vitro and in vivo experiments show that Cy@ZIF-8 nanoparticles not only have good PTT efficiency, but also possess remarkable NIR imaging capacity (Fig. 14A). ${ }^{72}$

The photothermal nanoparticles were also developed via covalent assembly process. Thus, the covalent reaction between dipeptides and iridoids together with the in situ self-assembly can form nanoparticles with tunable size distribution and good biocompatibility (Fig. 14B). Most importantly, these

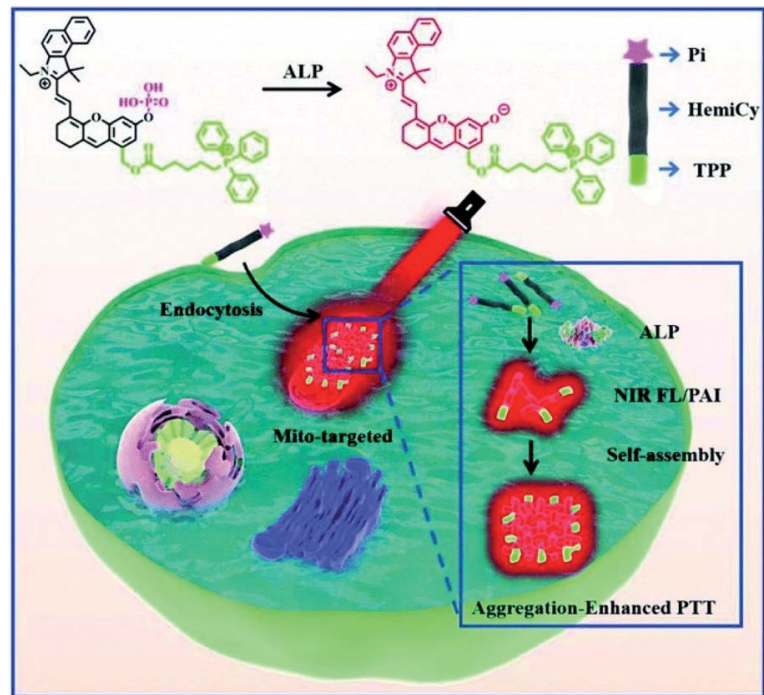

Fig. 13 Alkaline phosphatase (ALP) activatable and mitochondriatargeted probe for prostate cancer-specific bimodal imaging and aggregation-enhanced $\mathrm{PTT}^{71}$ (this figure has been reproduced from ref. 71 with permission from Royal Society of Chemistry).

nanoparticles have broad absorption ranging from UV to the near-infrared, high photothermal conversion efficiency and good photostability. Therefore, these nanoparticles are efficient in vivo tumor accumulation and selective tumor ablation. ${ }^{73}$

Based on supramolecular co-assembly, combined therapy with PTT has been developed. Luan et al. modified the photothermal dye (IR820) by lactosylation (LA-IR820). The coassembly of LA-IR820 with doxorubicin hydrochloride (DOX),

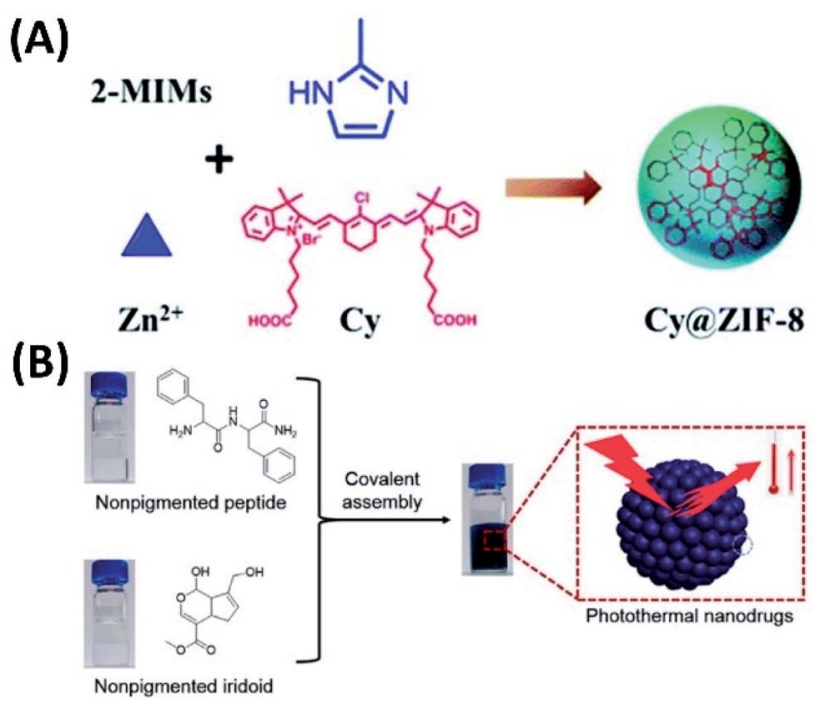

Fig. 14 (A) The NIR dye cyanine (Cy) was embedded into the zeolitic imidazolate framework-8 to form nanoparticles (Cy@ZIF-8) for NIR imaging-guided PTT; ${ }^{72}$ (this figure has been reproduced from ref. 72 with permission from Royal Society of Chemistry). (B) Covalent assembly of nonpigmented biomolecules for PTT; ${ }^{73}$ (this figure has been reproduced from ref. 73 with permission from American Chemical Society). 


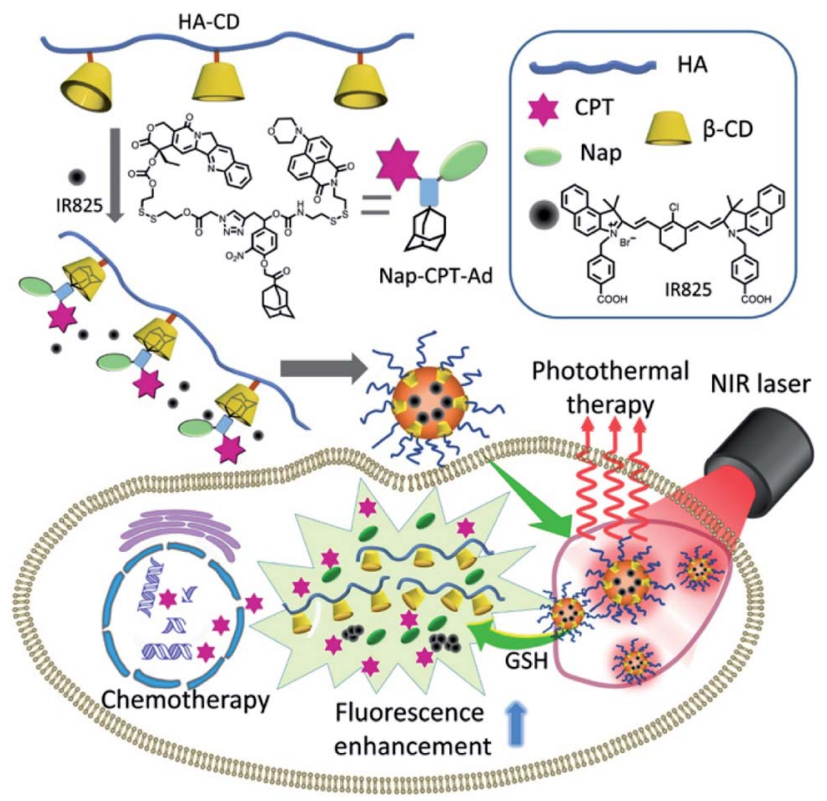

Fig. 15 IR825 dye loaded reduction-responsive and fluorescenceenhanced supramolecular nanoparticles for combinational photothermal chemotherapy of cancer ${ }^{77}$ (this figure has been reproduced from ref. 77 with permission from Elsevier).

which is chemotherapeutic drug, can form nanoparticles for synchronous delivery and synergistic targeting of IR820 and DOX as a combined PTT and chemotherapy ${ }^{74}$ Imaging-guided chemo-photothermal synergistic cancer therapy was also developed by the co-assembly of doxorubicin (DOX), photothermal compound indocyanine green (ICG) and amphiphilic polymer TPGS, which is the derivative of vitamin E. TPGS not only can be drug carrier, but also could serve as inhibitor of $P$ glycoprotein. Based on this type of co-assembly (T/DOX-ICG), combination cancer therapy can be achieved. ${ }^{75}$

For treating neuroblastoma, a strategy "photothermal immunotherapy" was developed by Fernandes et al. Thus, antiCTLA-4 checkpoint inhibition was also performed while PTT based on self-assembled Prussian blue nanoparticle (PBNP) was carrying out. For neuroblastoma animal model, photothermal immunotherapy results in obvious long-term surviving. ${ }^{76}$

The combined photothermal-chemotherapy based on hostguest interactions was developed by Zhao et al. In this system, hyaluronic acid was modified with $\beta$-cyclodextrin. The chemotherapy drug camptothecin was conjugated with adamantane and dye via the linkers containing embedded disulfide bond. The host-guest interactions between $\beta$-cyclodextrin and adamantane forms co-assemblies, which were further loaded with near-infrared absorbing dye IR825. When the system was included into cancer cell, disulfide bond could be cleaved by GHS, the release of camptothecin and dye IR825 for chemotherapy as well as PTT can be achieved, respectively. Moreover, the release of naphthalimide ensured fluorescence images. For the therapy on tumor-bearing mice by this conjugated system, significant tumor regression was observed (Fig. 15). ${ }^{77}$

Indoleamine 2,3-dioxygenase (IDO) is enzyme which can inhibit the activity of $\mathrm{T}$ lymphocyte and induce immune

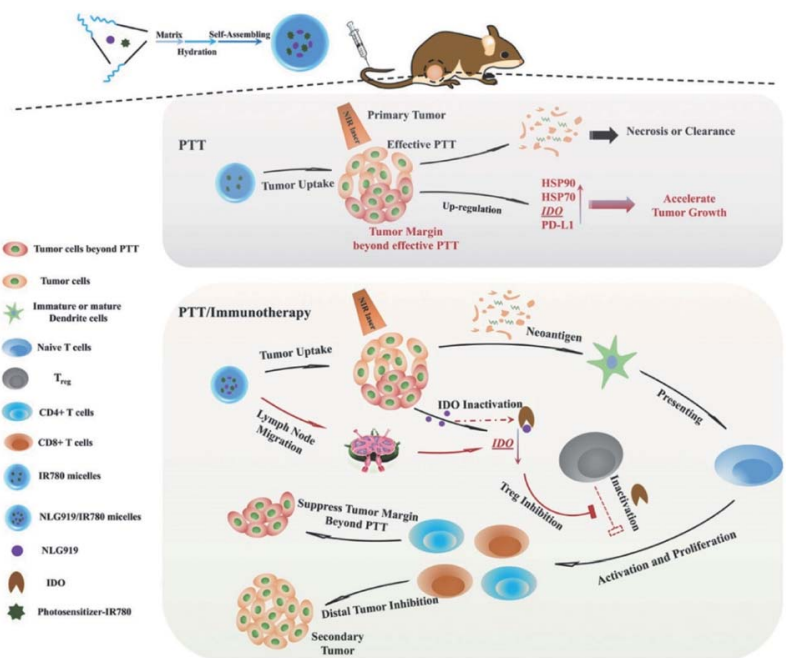

Fig. 16 Co-assembled amphipathic polymer MPEG-PCL with NLG919 and near-infrared absorbing dye IR780 formed micelles for combined PTT and immunotherapy ${ }^{78}$ (this figure has been reproduced from ref. 78 with permission from WILEY-VCH).

(A)

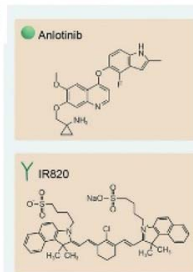

(B)

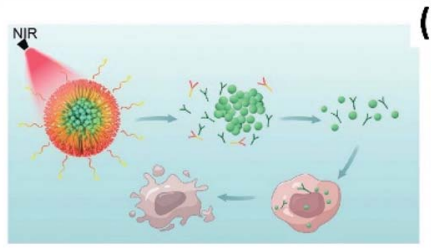

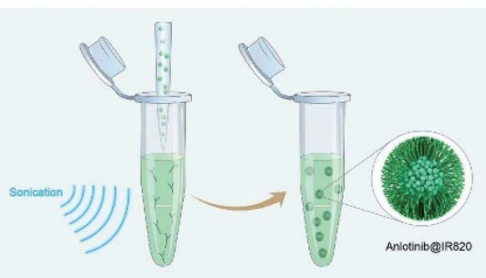

(C)

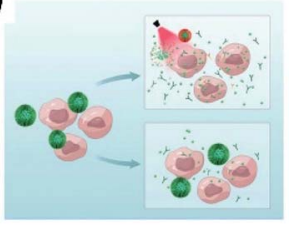

Fig. 17 (A) The co-assembly of anlotinib with NIR dye IR820 forms nanoparticles anlotinibaIR820 for synergistic PTT and targeted chemotherapy; (this figure has been reproduced from ref. 79 with permission from WILEY-VCH). (B and C) Under laser irradiation at 808 $\mathrm{nm}(\mathrm{B})$ or mild heating $(\mathrm{C})$ the photothermal-conversion and the disassembly of anlotinibaIR820 can be achieved, which induce the enhanced drug delivery ${ }^{79}$ (this figure has been reproduced from ref. 79 with permission from WILEY-VCH).

tolerance to cancer cells. Combined PTT and immunotherapy can be performed by releasing photosensitizer and IDO inhibitor NLG919, which is a small organic molecule. Qian et al. synthesized NLG919/IR780 micelles by co-assemble amphipathic polymer MPEG-PCL with NLG919 and near-infrared absorbing dye IR780. In vivo experiments show NLG/IR780 micelles can efficiently inhibit tumor growth and suppress the tumor margin (Fig. 16). ${ }^{78}$

Anlotinib is a novel targeted drug for cancer therapy. The coassembly of anlotinib with NIR dye IR820 forms nanoparticles anlotinib@IR820. Targeted therapy of breast cancer can be performed based on anlotinib@IR820, through synergistic PTT 
and targeted chemotherapy (Fig. 17A). For these systems, coassembly with IR820 increases water solvability of anlotinib. While photothermal effect promotes the release and uptake of anlotinib within cancer cell (Fig. 17B). Both in vitro and in vivo experiments systematically demonstrated that NIR-activated photothermal effect not only irradiated tumor directly but promoted the release and uptake of anlotinib, which strengthened the therapeutic efficacy synergistically. ${ }^{79}$

\section{Combined PDT and PTT depending on assembly}

The supramolecular assembly of some $\pi$-conjugated molecules can be used for both PTT and PDT. For example, the amphiphilic porphyrin molecules containing polyethylene glycol chains and pentafluorobenzene moieties were synthesized.
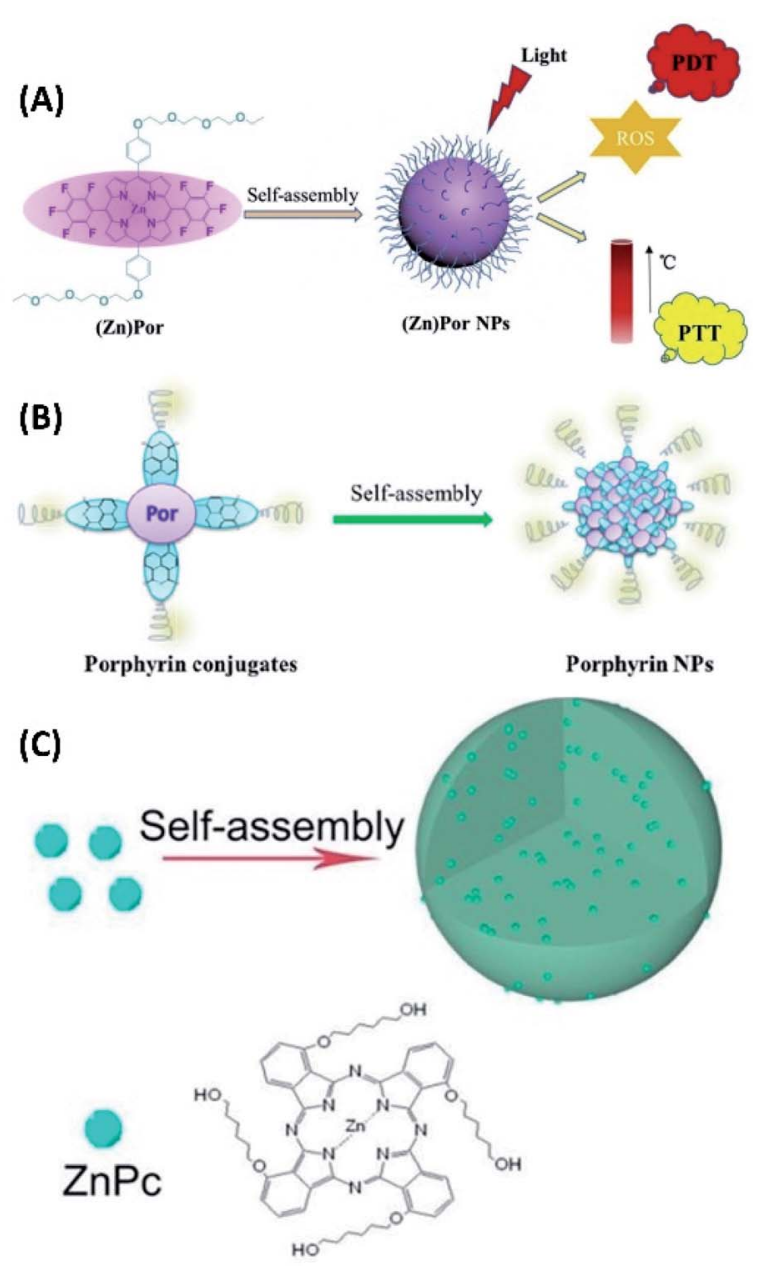

Fig. 18 (A) The self-assembly of amphiphilic fluoroporphyrins for synergistic PDT and PTT; 80 (this figure has been reproduced from ref. 80 with permission from Elsevier). (B) Self-assembled donoracceptor-donor porphyrin for PDT/PTT synergistic therapy: ${ }^{81}$ (this figure has been reproduced from ref. 81 with permission from Elsevier). (C) The self-assembly of zinc phthalocyanine for synergistic PDT and $\mathrm{PT}{ }^{82}$ (this figure has been reproduced from ref. 82 with permission from American Chemical Society).

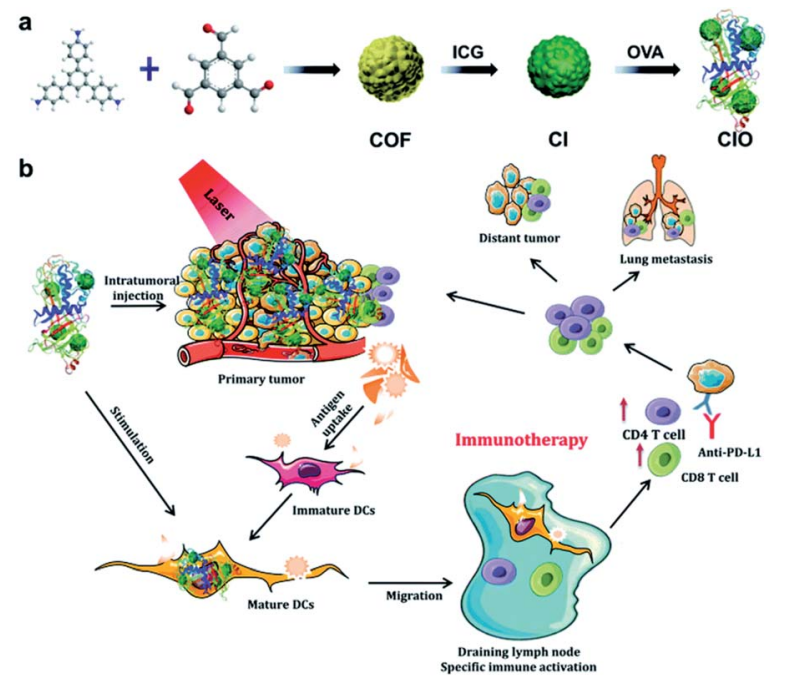

Fig. 19 The co-assembly of photosensitizers with COF showing combined PDT and PTT ${ }^{84}$ (this figure has been reproduced from ref. 84 with permission from Royal Society of Chemistry).

Both the self-assembly of zinc porphyrin (ZnPor) and free-base porphyrin (Por) can form nanoparticles (Fig. 18A), which increase the tumor targeting properties of porphyrins. Upon $635 \mathrm{~nm}$ laser irradiation, ZnPor nanoparticles could show higher ROS generation efficiency for PDT. However, the nanoparticles of free-base porphyrin exhibited better photothermal effect for PTT. ${ }^{\mathbf{8 0}}$ Another amphiphilic porphyrin compound (TPAPor) containing polyethylene glycol chains and triphenylamine moieties was developed by the same group. This donoracceptor-donor porphyrin has clear red-shift of absorption. The self-assembly of TPAPor can form nanoparticles with good biocompatibility (Fig. 18B). Upon light irradiation, TPAPor nanoparticles show excellent photothermal conversion efficiency and relatively low singlet oxygen generation. Therefore, TPAPor nanoparticles can be used for combined PTT/PDT cancer therapy. ${ }^{81}$ Another amphiphilic porphyrin with typical donor-acceptor structures can be the molecule containing naphthalimide and PEG chains (NI-Por). Both NI-Por and its Zinc complex (NI-ZnPor) can self-assemble in nanoparticles for performing PDT/PTT synergistic cancer therapy under light irradiation. $^{82}$

Yang et al. synthesized zinc phthalocyanine molecules containing alkyl chain and hydroxyl groups (ZnPc). The selfassembly of $\mathrm{ZnPc}$ can form nanoparticles with fluorescence (Fig. 17C). Therefore, ZnPc nanoparticles not only exhibit good photothermal properties, but also have the capability for the generation of singlet oxygen. For the cancer therapy of $\mathrm{ZnPc}$ nanoparticles, the dual-modal PTT and PDT can be achieved. ${ }^{83}$

The adsorption of photosensitizers onto covalent organic framework (COF) can create the system with combined PDT and PTT ability. Pang et al. synthesized the COF by using 1,3,5benzenetricarbaldehyde (BTCA) and 1,3,5-tris(4-aminophenyl) benzene (TAPB) as starting materials. The stepwise adsorption of indocyanine green (ICG) and chicken ovalbumin (OVA) onto the COF can form the conjugates COF@ICG@OVA, which has 


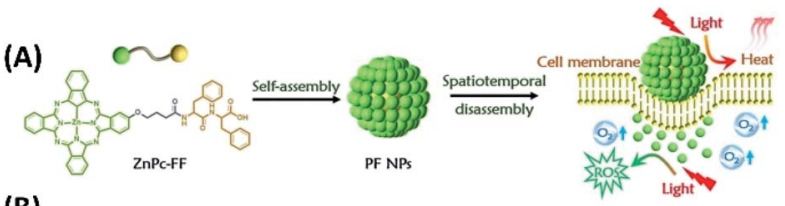

(B)

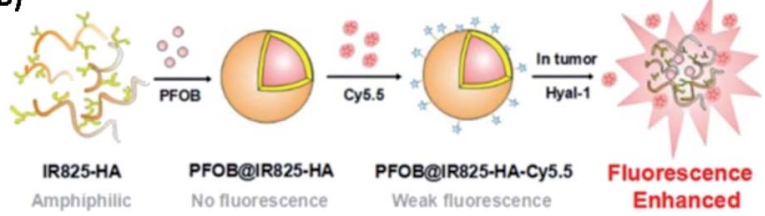

Fig. 20 (A) The self-assembly of phthalocyanine-peptide conjugate for adaptive tumor theranostics; ${ }^{86}$ (this figure has been reproduced from ref. 86 with permission from WILEY-VCH). (B) The co-assembly of hyaluronic acid (HA) with two near-infrared dyes (Cy5.5, IR825) and perfluorooctylbromide (PFOB) for enhanced fluorescence/CT/photoacoustic imaging guided $\mathrm{PTT}^{85}$ (this figure has been reproduced from ref. 85 with permission from Elsevier).

high photothermal conversion efficiency as well as the production of ROS upon light irradiation. This type of combinational PTT/PDT therapy, together with anti-PD-L1 checkpoint blockade therapy, can effectively eliminate primary tumors and inhibit the metastasis of cancer cells by generating strong immune responses (Fig. 19). ${ }^{84}$

The supramolecular assemblies, which were developed for PTT, can also be used as theranostic agent. For example, the enhanced fluorescence/CT/photoacoustic imaging guided PTT has been studied by Liang and Wang et al. They prepared multifunctional nanoparticles (PFOB@IR825-HA-Cy5.5) based on the co-assembly of hyaluronic acid (HA) with two nearinfrared dyes (Cy5.5, IR825) and perfluorooctylbromide (PFOB) (Fig. 20B). Since PFOB can absorb X-rays, while Cy5.5 has fluorescence, the triple-modal imaging including fluorescence, CT and PAT can achieved when PFOB@IR825-HA-Cy5.5 nanoparticles were disassembled by hyaluronidases (Hyals) in the tumor. The near-infrared dye IR825 within the assembly would act as photothermal agent for achieving effective tumor ablation upon infrared laser irradiation. ${ }^{85}$

The nanoparticles with switchable multimodal theranostics were developed from the supramolecular assemblies of a phthalocyanine-peptide conjugate (PF). Depending on interactions between cell membrane and PF nanoparticles, the photoactivity of the PF nanoparticles can be changed from PTT and photoacoustic imaging to PDT and fluorescence imaging (Fig. 20A). ${ }^{86}$

\section{Conclusions}

In summary, the PDT and PTT based on assembled small organic molecules have developed greatly in the past few years. For the application of PDT and PTT, the ideas of supramolecular assembly do bring great advantages, which include the better biological compatibility, controlled release of drug and photosensitizers, better photophysical properties of photosensitizers, as well as promoted photothermal efficiency. Depending on the hierarchically supramolecular assembly, the efficiency of the systems for PDT and PTT can be greatly increased. Moreover, the synergy of different treatments, such as combined chemotherapy with PDT, also can be easily achieved upon supramolecular assembly. In the future, the larger development of the research in this field are expected, due to the flexibility, diversity, and regulatory of supramolecular systems.

\section{Conflicts of interest}

There are no conflicts to declare.

\section{Acknowledgements}

The authors gratefully acknowledge support for this work from the Natural Science Foundation of China (no. 21631003, 21671017, 21773006, 22072004), Major Science and Technology Program for Water Pollution Control and Treatment (2017ZX07402001), Beijing Municipal Commission of Education, and University of Science and Technology Beijing.

\section{Notes and references}

1 S. J. Henley, C. C. Thomas, H. K. Weir, M. Wu, V. B. Benard, L. C. Richardson, E. M. Ward, R. L. Sherman, B. A. Kohler, S. Scott, D. R. Lewis, K. Cronin, J. Ma, F. Islami, A. Jemal, R. N. Anderson and A. U. Firth, Cancer, 2020, 126, 2225-2249.

2 H. Abrahamse and M. R. Hamblin, Biochem. J., 2016, 473, 347-364.

3 S. Gai, G. Yang, P. Yang, F. He, J. Lin, D. Jin and B. Xing, Nano Today, 2018, 19, 146-187.

4 S. S. Lucky, K. C. Soo and Y. Zhang, Chem. Rev., 2015, 115, 1990-2042.

5 X. Ma and Y. Zhao, Chem. Rev., 2015, 115, 7794-7839.

6 S. Singh, A. Aggarwal, N. V. S. D. K. Bhupathiraju, G. Arianna, K. Tiwari and C. M. Drain, Chem. Rev., 2015, 115, 1026110306.

7 Z. Zhou, J. Song, L. Nie and X. Chen, Chem. Soc. Rev., 2016, 45, 6597-6626.

8 K.-T. Li, Q. Chen, D.-W. Wang, Q.-Q. Duan, S. Tian, J.-W. He, Y.-S. Ou and D.-Q. Bai, Cancer Med., 2016, 5, 3186-3193.

9 J. Yue, Y. Shen, L. Liang, L. Cong, W. Xu, W. Shi, C. Liang and S. Xu, Anal. Chem., 2020, 92, 6081-6087.

10 H. Abrahamse and M. R. Hamblin, Biochem. J., 2016, 473, 347-364.

11 F. Heinemann, J. Karges and G. Gasser, Acc. Chem. Res., 2017, 50, 2727-2736.

12 E. S. Nyman and P. H. Hynninen, J. Photochem. Photobiol., B, 2004, 73, 1-28.

13 Y. Nosaka and A. Y. Nosaka, Chem. Rev., 2017, 117, 1130211336.

14 A. L. Harris, Nat. Rev. Cancer, 2002, 2, 38-47.

15 R. A. Gatenby and R. J. Gillies, Nat. Rev. Cancer, 2004, 4, 891899.

16 A. P. Castano, P. Mroz and M. R. Hamblin, Nat. Rev. Cancer, 2006, 6, 535-545. 
17 S. O. Gollnick and C. M. Brackett, Immunol. Res., 2010, 46, 216-226.

18 A. D. Garg and P. Agostinis, Photochem. Photobiol. Sci., 2014, 13, 474-487.

19 J. M. Dabrowski and L. G. Arnaut, Photochem. Photobiol. Sci., 2015, 14, 1765-1780.

20 M. Karimi, P. Sahandi Zangabad, S. Baghaee-Ravari, M. Ghazadeh, H. Mirshekari and M. R. Hamblin, J. Am. Chem. Soc., 2017, 139, 4584-4610.

21 T. L. Doane and C. Burda, Chem. Soc. Rev., 2012, 41, 28852911.

22 A. Master, M. Livingston and A. Sen Gupta, J. Controlled Release, 2013, 168, 88-102.

23 Z. Zhou, J. Song, L. Nie and X. Chen, Chem. Soc. Rev., 2016, 45, 6597-6626.

24 M. Cao, R. Xing, R. Chang, Y. Wang and X. Yan, Coord. Chem. Rev., 2019, 397, 14-27.

25 S. Li, W. Zhang, H. Xue, R. Xing and X. Yan, Chem. Sci., 2020, 11, 8644-8656.

26 N. Cheng, Y. Chen, J. Yu, J.-j. Li and Y. Liu, Bioconjugate Chem., 2018, 29, 1829-1833.

27 X. Dai, X. Dong, Z. Liu, G. Liu and Y. Liu, Biomacromolecules, 2020, 21, 5369-5379.

28 X. Li, S. Lee and J. Yoon, Chem. Soc. Rev., 2018, 47, 11741188.

29 Z. Wang, C. J. Medforth and J. A. Shelnutt, J. Am. Chem. Soc., 2004, 126, 15954-15955.

30 G. Bottari, O. Trukhina, M. Ince and T. Torres, Coord. Chem. Rev., 2012, 256, 2453-2477.

31 N. K. Shee, M. K. Kim and H.-J. Kim, Nanomaterials, 2020, 10, 2314.

32 J. P. McEvoy and G. W. Brudvig, Chem. Rev., 2006, 106, 44554483.

33 G. D. Scholes, G. R. Fleming, A. Olaya-Castro and R. van Grondelle, Nat. Chem., 2011, 3, 763-774.

34 I. G. Denisov, T. M. Makris, S. G. Sligar and I. Schlichting, Chem. Rev., 2005, 105, 2253-2277.

35 Y.-L. P. Ow, D. R. Green, Z. Hao and T. W. Mak, Nat. Rev. Mol. Cell Biol., 2008, 9, 532-542.

36 H.-R. Jia, Y.-W. Jiang, Y.-X. Zhu, Y.-H. Li, H.-Y. Wang, X. Han, Z.-W. Yu, N. Gu, P. Liu, Z. Chen and F.-G. Wu, J. Controlled Release, 2017, 255, 231-241.

37 C. Ji, Q. Gao, X. Dong, W. Yin, Z. Gu, Z. Gan, Y. Zhao and M. Yin, Angew. Chem., Int. Ed., 2018, 57, 11384-11388.

38 Z. Xie, X. Cai, C. Sun, S. Liang, S. Shao, S. Huang, Z. Cheng, M. Pang, B. Xing, A. A. A. Kheraif and J. Lin, Chem. Mater., 2019, 31, 483-490.

39 F. J. M. Hoeben, P. Jonkheijm, E. W. Meijer and A. P. H. J. Schenning, Chem. Rev., 2005, 105, 1491-1546.

40 T. Aida, E. W. Meijer and S. I. Stupp, Science, 2012, 335, 813817.

41 G. Ouyang, L. Ji, Y. Jiang, F. Wurthner and M. Liu, Nat. Commun., 2020, 11, 5910.

42 L. Cheng, C. Wang, L. Feng, K. Yang and Z. Liu, Chem. Rev., 2014, 114, 10869-10939.

43 Y. Liu, P. Bhattarai, Z. Dai and X. Chen, Chem. Soc. Rev., 2019, 48, 2053-2108.
44 L. Dykman and N. Khlebtsov, Chem. Soc. Rev., 2012, 41, 2256-2282.

45 K. Yang, L. Feng, X. Shi and Z. Liu, Chem. Soc. Rev., 2013, 42, 530-547.

46 T. Liu, C. Wang, X. Gu, H. Gong, L. Cheng, X. Shi, L. Feng, B. Sun and Z. Liu, Adv. Mater., 2014, 26, 3433-3440.

47 X. Wang, P. Wang, S. Xue, X. Zheng, Z. Xie, G. Chen and T. Sun, Org. Biomol. Chem., 2018, 16, 1591-1597.

48 C. Wang, L. Liu, H. Cao and W. Zhang, Biomater. Sci., 2017, 5, 274-284.

49 Y.-J. Kim, H.-I. Lee, J.-K. Kim, C.-H. Kim and Y.-J. Kim, Colloids Surf., B, 2020, 189, 110829.

50 C. Luo, B. Sun, C. Wang, X. Zhang, Y. Chen, Q. Chen, H. Yu, H. Zhao, M. Sun, Z. Li, H. Zhang, Q. Kan, Y. Wang, Z. He and J. Sun, J. Controlled Release, 2019, 302, 79-89.

51 W. Song, J. Kuang, C.-X. Li, M. Zhang, D. Zheng, X. Zeng, C. Liu and X.-Z. Zhang, ACS Nano, 2018, 12, 1978-1989.

52 H. Cheng, G.-L. Fan, J.-H. Fan, R.-R. Zheng, L.-P. Zhao, P. Yuan, X.-Y. Zhao, X.-Y. Yu and S.-Y. Li, Macromol. Biosci., 2019, 19, 1800410.

53 H. Cheng, G.-L. Fan, J.-H. Fan, L.-P. Zhao, R.-R. Zheng, X.-Y. Yu and S.-Y. Li, Nanoscale, 2019, 11, 9008-9014.

54 M. Li, Y. Ning, J. Chen, X. Duan, N. Song, D. Ding, X. Su and Z. Yu, Nano Lett., 2019, 19, 7965-7976.

55 P. Semeraro, G. Chimienti, E. Altamura, P. Fini, V. Rizzi and P. Cosma, Mater. Sci. Eng., C, 2018, 85, 47-56.

56 J. Li, A. Wang, L. Zhao, Q. Dong, M. Wang, H. Xu, X. Yan and S. Bai, ACS Appl. Mater. Interfaces, 2018, 10, 28420-28427.

57 J. Li, A. Wang, P. Ren, X. Yan and S. Bai, Chem. Commun., 2019, 55, 3191-3194.

58 Q. Zou, R. Chang, R. Xing, C. Yuan and X. Yan, J. Controlled Release, 2020, 319, 344-351.

59 J. Cheng, H. Zhao, L. Yao, Y. Li, B. Qi, J. Wang and X. Yang, ACS Appl. Mater. Interfaces, 2019, 11, 29498-29511.

60 Z. Zhang, R. Wang, X. Huang, R. Luo, J. Xue, J. Gao, W. Liu, F. Liu, F. Feng and W. Qu, ACS Appl. Mater. Interfaces, 2020, 12, 5680-5694.

61 L. Xu, M. Zhao, Y. Yang, Y. Liang, C. Sun, W. Gao, S. Li, B. He and Y. Pu, J. Mater. Chem. B, 2017, 5, 9157-9164.

62 W. Mao, Y. Liao and D. Ma, Chem. Commun., 2020, 56, 41924195.

63 R. Zhang, R. Xing, T. Jiao, K. Ma, C. Chen, G. Ma and X. Yan, ACS Appl. Mater. Interfaces, 2016, 8, 13262-13269.

64 L. Liu, Y. Bao, J. Wang, C. Xiao and L. Chen, Dyes Pigm., 2020, 177, 107922.

65 X. Sun, Z. Cao, K. Mao, C. Wu, H. Chen, J. Wang, X. Wang, X. Cong, Y. Li, X. Meng, X. Yang, Y.-G. Yang and T. Sun, Biomaterials, 2020, 240, 119845.

66 H. Zhang, K. Liu, S. Li, X. Xin, S. Yuan, G. Ma and X. Yan, ACS Nano, 2018, 12, 8266-8276.

67 H. Zhang, S. Bo, K. Zeng, J. Wang, Y. Li, Z. Yang, X. Zhou, S. Chen and Z.-X. Jiang, J. Mater. Chem. B, 2020, 8, 44694474.

68 R. Zagami, G. Sortino, E. Caruso, M. C. Malacarne, S. Banfi, S. Patanè, L. Monsù Scolaro and A. Mazzaglia, Langmuir, 2018, 34, 8639-8651. 
69 H. Wang, W. Zhao, X. Liu, S. Wang and Y. Wang, ACS Appl. Bio Mater., 2020, 3, 593-601.

70 Q. Zou, M. Abbas, L. Zhao, S. Li, G. Shen and X. Yan, J. Am. Chem. Soc., 2017, 139, 1921-1927.

71 D. Yao, S. Yang, Y. Wang, K. Bian, W. Yang, D. Wang and B. Zhang, Nanoscale, 2019, 11, 6307-6314.

72 Y. Li, N. Xu, J. Zhou, W. Zhu, L. Li, M. Dong, H. Yu, L. Wang, W. Liu and Z. Xie, Biomater. Sci., 2018, 6, 2918-2924.

73 Y. Liu, G. Shen, L. Zhao, Q. Zou, T. Jiao and X. Yan, ACS Appl. Mater. Interfaces, 2019, 11, 41898-41905.

74 Y. Jiang, C. Huang and Y. Luan, Int. J. Nanomed., 2020, 15, 4431-4440.

75 H. Gao, Y. Bai, L. Chen, G. E. Fakhri and M. Wang, Int. J. Nanomed., 2020, 15, 809-819.

76 J. Cano-Mejia, R. A. Burga, E. E. Sweeney, J. P. Fisher, C. M. Bollard, A. D. Sandler, C. R. Y. Cruz and R. Fernandes, Nanomedicine, 2017, 13, 771-781.

77 Y. Zhang, D. Yang, H. Chen, W. Q. Lim, F. S. Z. Phua, G. An, P. Yang and Y. Zhao, Biomaterials, 2018, 163, 14-24.
78 J. Peng, Y. Xiao, Y. Jia, Y. Qu, Z. Qian, W. Li, L. Tan and Q. Yang, Adv. Sci., 2018, 5, 1700891.

79 C. He, L. Yu, L. Ding, Y. Chen and Y. Hao, Adv. Funct. Mater., 2020, 30, 1908907.

80 L. Yang, H. Li, D. Liu, H. Su, K. Wang, G. Liu, X. Luo and F. Wu, Colloids Surf., B, 2019, 182, 110345.

81 L. Yang, J. Zhou, Z. Wang, H. Li, K. Wang, H. Liu and F. Wu, Dyes Pigm., 2020, 182, 108664.

82 M. Yang, S. Cao, X. Sun, H. Su, H. Li, G. Liu, X. Luo and F. Wu, Bioconjugate Chem., 2020, 31, 663-672.

83 Z. Wang, S. Gai, C. Wang, G. Yang, C. Zhong, Y. Dai, F. He, D. Yang and P. Yang, Chem. Eng. J., 2019, 361, 117-128.

84 Y. Zhou, S. Liu, C. Hu, L. Cai and M. Pang, J. Mater. Chem. B, 2020, 8, 5451-5459.

85 X. Liang, L. Fang, X. Li, X. Zhang and F. Wang, Biomaterials, 2017, 132, 72-84.

86 S. Li, L. Zhao, R. Chang, R. Xing and X. Yan, Chem.-Eur. J., 2019, 25, 13429-13435. 